\title{
Prevalence of Post-Traumatic Stress Disorder Caused by War in Veterans
}

\section{ART ICLE INF O}

\section{Article Type}

Descriptive Study

\section{Authors}

Rahnejat A.M. ${ }^{1} P h D$

Dabagi P.* $P h D$,

Rabiei M. ${ }^{2} P h D$,

Taghva $A .^{3} M D$,

Valipoor H. ${ }^{1} M S C$

Donyavi V. $M D$,

Ebrahimi M.R. ${ }^{3} M D$

How to cite this article
Rahnejat AM, Dabagi P, Rabiei M,
Taghva A, Valipoor H, Donyavi V,
Ebrahimi MR. Prevalence of Post-
Traumatic Stress Disorder Caused
by War in Veterans. Iranian Journal
of War \& Public Health. 2017;9(1):
15-23.

\section{A B S T R A C T}

Aims The post-traumatic stress disorders sometimes continue years after wars. The aim of the study was to estimate the prevalence of post-traumatic stress disorders in the veterans 26 years after the end of Iran-Iraq war.

Instrument \& Methods In this cross-sectional study, 341 Iranian veterans were studied in 2014. The subjects were selected via cluster random sampling method. Data was collected using post-traumatic stress disorder checklist-military edition (PCL-M-5), clinician-administered post-traumatic stress disorders scale for DSM-5 (CAPS-5), and a demographic questionnaire. Data was analyzed through descriptive statistics and Pearson correlation coefficient and simultaneous regression analysis tests.

Findings 82 veterans (24.0\%) were with post-traumatic stress disorders diagnostic criteria. In addition, their most prevalent post-traumatic stress disorders symptoms were arousal and reactivity, and negative changes in the cognition and mood, successively. Domographic parameters, including educational level ( $\mathrm{r}=-0.191 ; \mathrm{p}<0.01)$, age during military services $(\mathrm{r}=0.046 ; \mathrm{p}<0.05)$, military services length $(\mathrm{r}=0.107 ; \mathrm{p}<0.05)$, and injury and its severity $(\mathrm{r}=0.347 ; \mathrm{p}<0.01)$ significantly correlated with post-traumatic stress disorders. In addition, $13 \%$ of the post-traumatic stress disorders variance were explained by the demographic parameters $(\mathrm{p}<0.01)$.

Conclusion 26 years after the end of Iran-Iraq war, many veterans are with post-traumatic stress disorders, and their most prevalent symptom is arousal and reactivity.

\section{Keywords Stress Disorder, Post-Traumatic; Prevalence; War; Veterans}

\section{I T A T I O N L IN KS}

[1] Traumatic events and posttraumatic ... [2] Treating trauma survivors... [3] Causal metacognitive model war-related chronic... [4] Review and analysis of the Metacognitive... [5] Comparative effectiveness of ... [6] Prevalence of post-traumatic stress... [7] Posttraumatic stress disorder in the ... [8] Trauma and the vietnam war ... [9] Population attributable fractions of ... [10] PTSD after deployment to Iraq... [11] Haunted by combat: Understanding ... [12] Military psychol: Clinical and ... [13] Flashback: Post traumatic stress ... [14] Cognitive behavior therapy of post... [15] Bringing the war back home: mental health ... [16] PTSD prevalence, associated exposures ... [17] Comorbidity of PTSD and depression in ... [18] Combat duty in Iraq and Afghanistan ... [19] Epidemiological study of psychological ... [20] Trends and risk factors for mental... [21] Invisible woPTSD symptom increases in ... [22] PTSD symptom increases ... [23] Prevalence of mental health ... [24] The psychological risks of Vietnam ... [25] Ethnic differences in posttraumatic ... [26] The prevalence of common mental ... [27] The prevalence of ptsd in conscript ... [28] Study of sleep quality in chemical-warfare ... [29] The role of thought control ... [30] Psychometric properties of the ... [31] The prevalence of post-traumatic ... [32] Posttraumatic stress disorder in DSM-5: New criteria ... [33] Normalization of Clinician Administered PTSD ... [34] Treatment for posttraumatic stress disorder ... [35] Health status of Vietnam ... [36] A longitudinal and retrospective ... [37] Prevalence estimates of combat-related PTSD ... [38] Posttraumatic stress disorder and posttraumatic ... [39] Post-traumatic stress disorder and chronic ... [40] The health of UK military personnel .. [41] Prevalence of psychotic co morbidity ... [42] Conceptual and practical principles ... [43] Meta cognitive therapy ... [44] Met cognitive therapy for PTSD ... [45] Chronic PTSD Treated with Meta ... [46] Understanding the relationship ... [47] The Thought Control Questionnaire ... [48] Behavioral formulation of posttraumatic ... [49] Cognitive reserve in neuropsychiatry ... [50] Intelligence and other predisposing ...

\section{Article History}

Received: June 29, 2016

Accepted: November 21, 2016

ePublished: March 10, 2017 


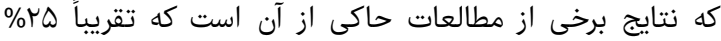

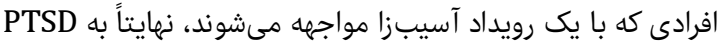

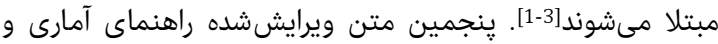

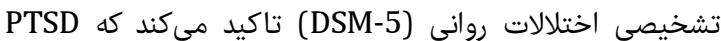

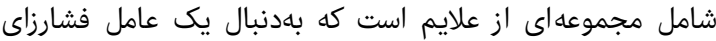

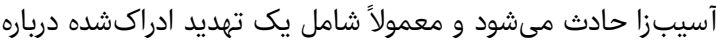

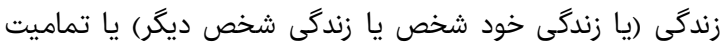

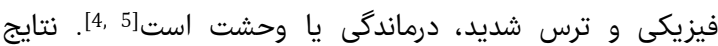

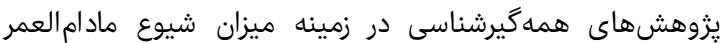

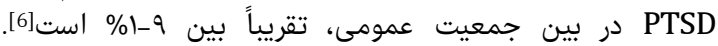

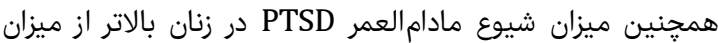

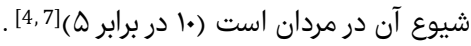

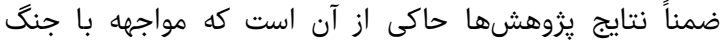

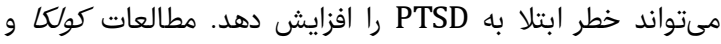

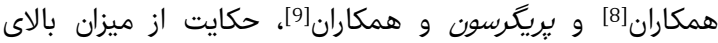

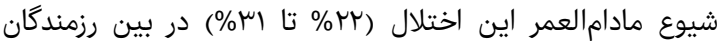

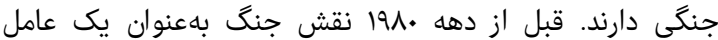

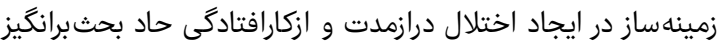

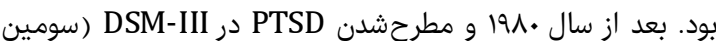

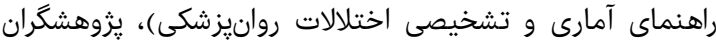

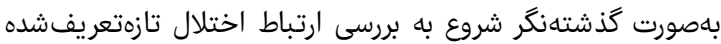

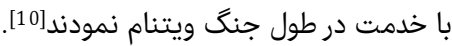

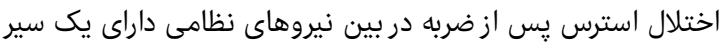

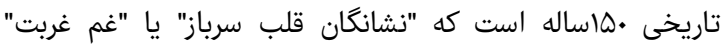

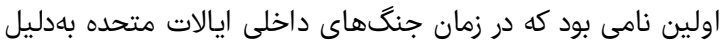

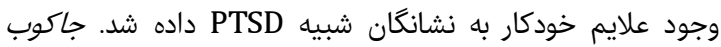

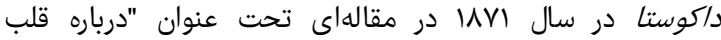

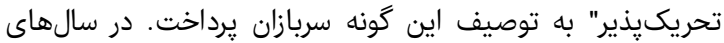

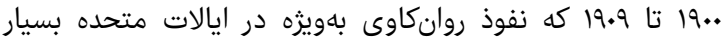

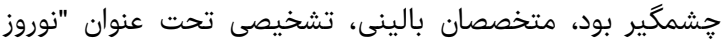

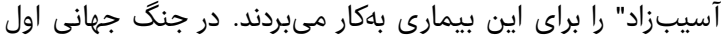

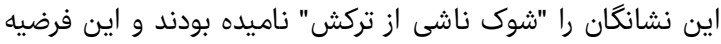

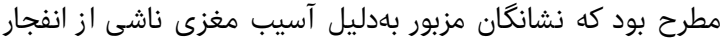

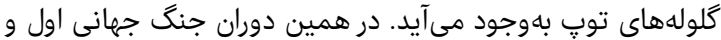

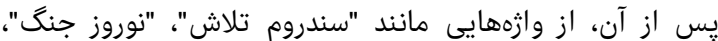

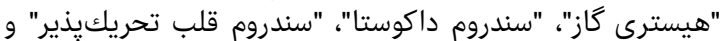

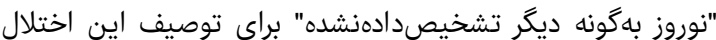

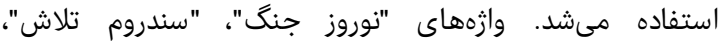

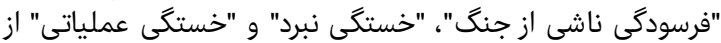

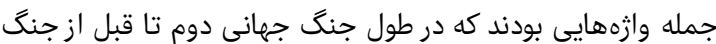

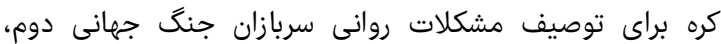

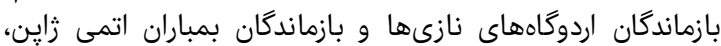

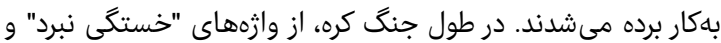

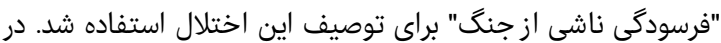

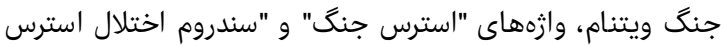

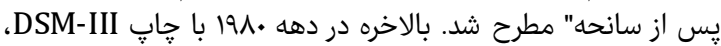

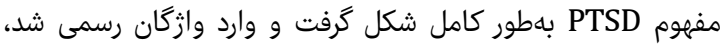

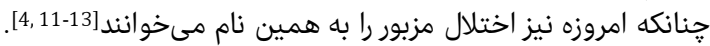

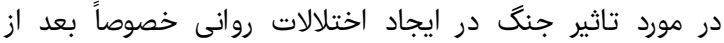

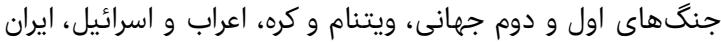

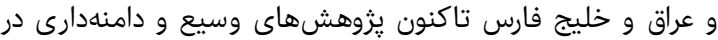
كشورهاى مختلف در مورد نظاميان و شركتكنندًان دران در جنگ انجام داري

دوره 9، شماره ا، زمستان هوس|
ميزان شيوع اختلال استرس يس از ضربه ناشى از

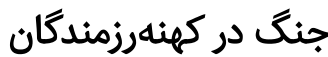

PhD ميرمحسن راهنجات كروه روانشناسى بالينى، دانشكده يزشكى، دانشخاه علوم يزشكى آجا، تهران، ايران

PhD "يرويز دباغى كَروه روانشناسى بالينى، دانشكده يزشكى، دانشكاه علوم يزشكى آجا، تهران، ايران

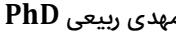
كروه روانشناسى بالينى، دانشكده يزشكى، دانشكاه علوم يزشكى بقيه|...(ه)،

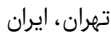

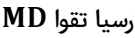

كروه روانيزشكى، دانشكده يزشكى، دانشكاه علوم يزشكى آجا، تهران، ايران

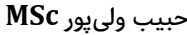

كروه روانشناسى بالينى، دانشكده يزشكى، دانشكاه علوم يزشكى آجا، تهران، ايران

وحيد دنيوى ايرى

كروه روانيزشكى، دانشكده يزشكى، دانشكاه علوم يزشكى آجا، تهران، ايران

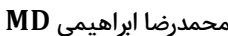

كَروه روانيزشكى، دانشكده يزشكى، دانشكاه علوم يزشكى آجا، تهران، ايران

جكيده

اهداف: يزوهشها نشان دادهاند عوارض روانى جنگ مانند اختلال

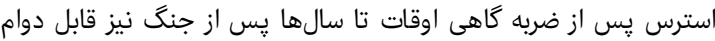

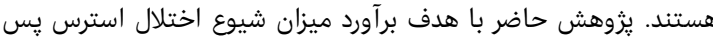

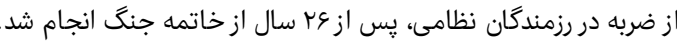

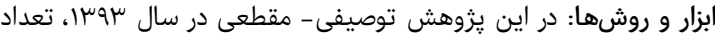

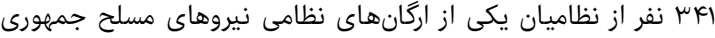

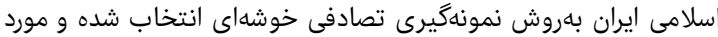

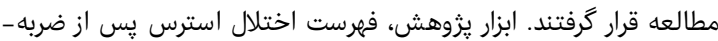

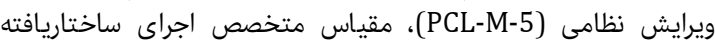

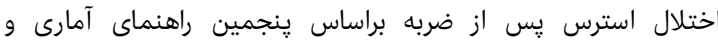

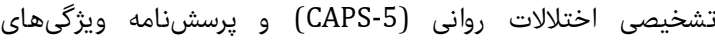

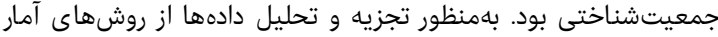

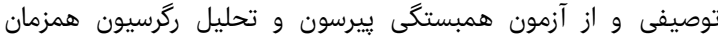

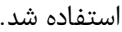

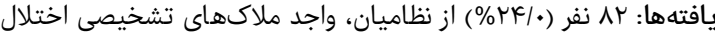

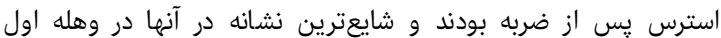

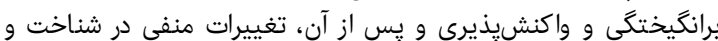

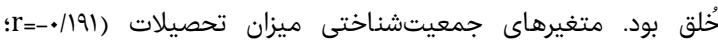

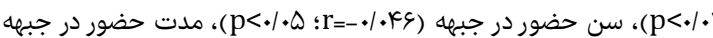

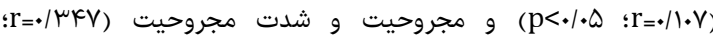

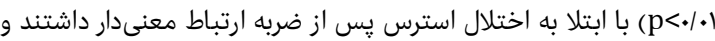

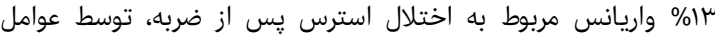

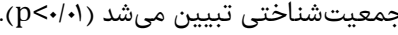

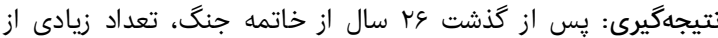

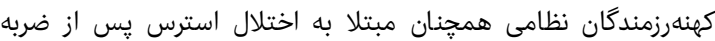

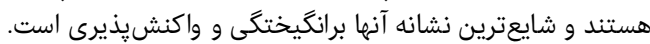

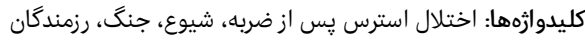

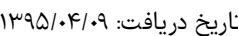

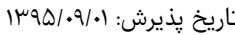

"نويسنده مسئول: dabaghi_44@yahoo.com

مقدمه - مقد

يكى از اختلالات روان شناختى كه در مواجهه با رويدادهاى آسيبزا

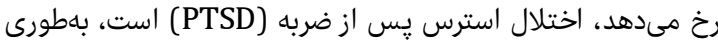




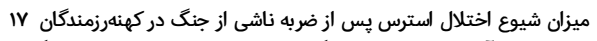

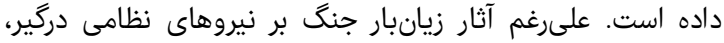

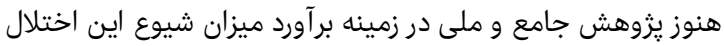

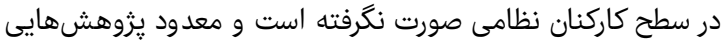

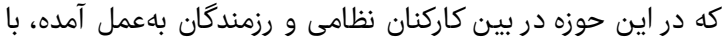

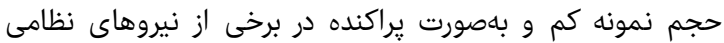

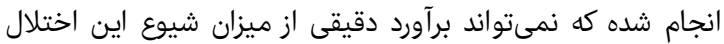

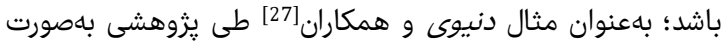

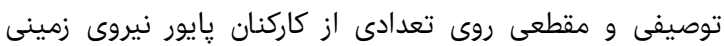

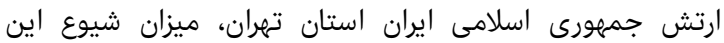

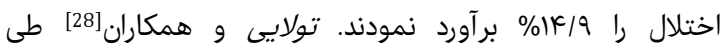
مطالعهاى در زمينه سلامت روانى مجروحان شيميايى سردشت،

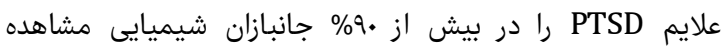

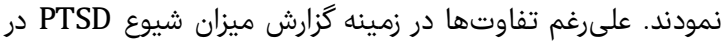

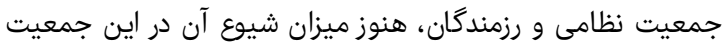

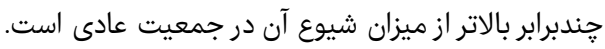

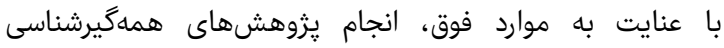

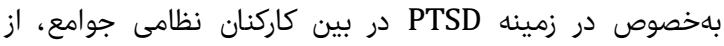

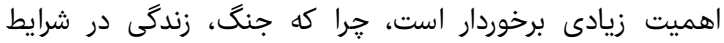

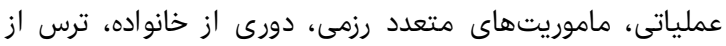

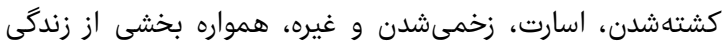

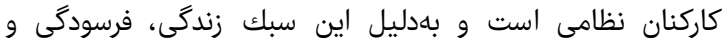

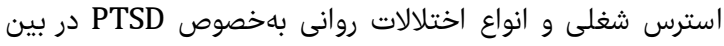

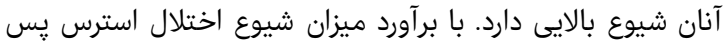

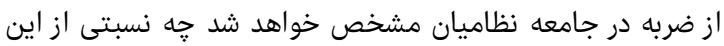

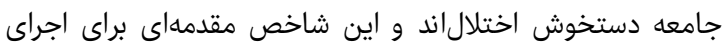

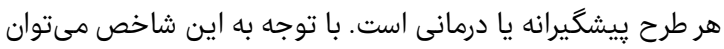

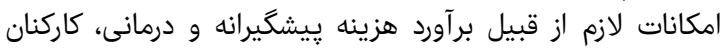

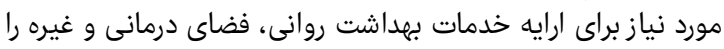

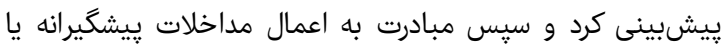

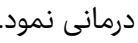
بنابراين يزوهش حاضر با هود درف برآورد ميزان شيوع اختلال استرس

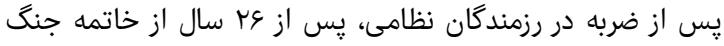

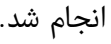

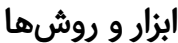

يزوهش حاضر، ازنوع مطالعات توصيفى و همهگيرشناسى است كه

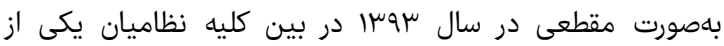

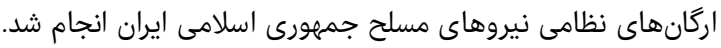

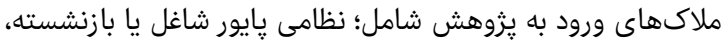

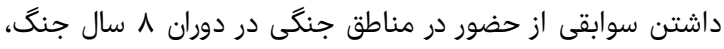

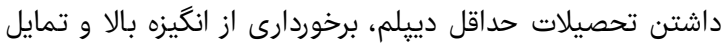

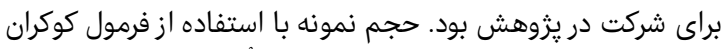

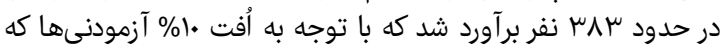

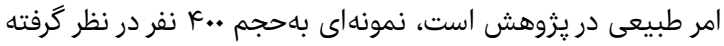

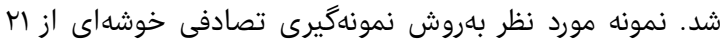

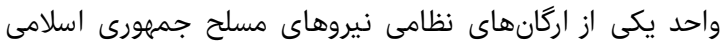

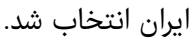

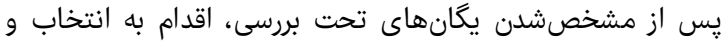

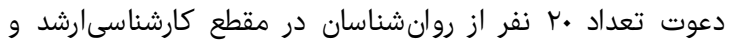

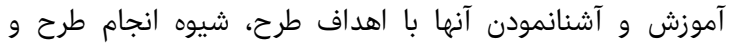

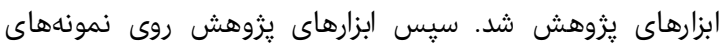

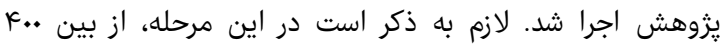

كرفته است كه همخى نشاندهنده تاثيرات مخرب جنَ و حضور در

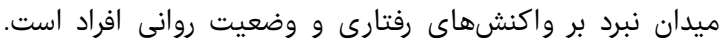

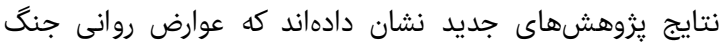

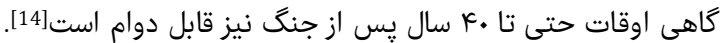

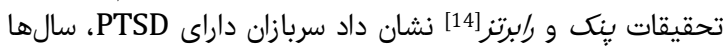

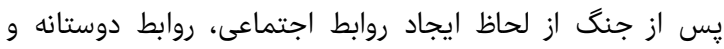

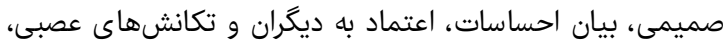

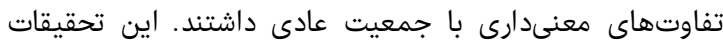

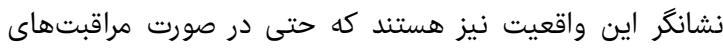

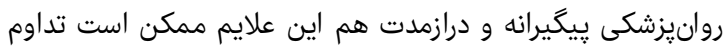
يابند.

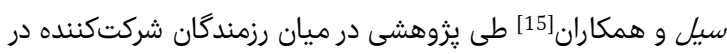

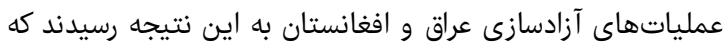

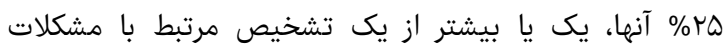

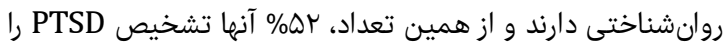

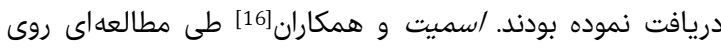

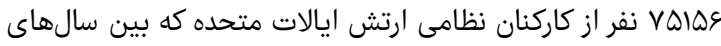

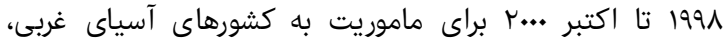

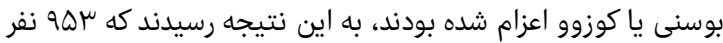

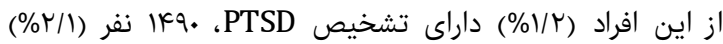

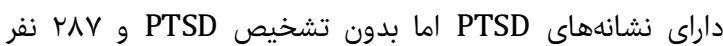

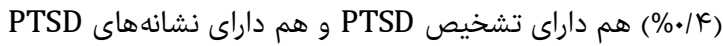

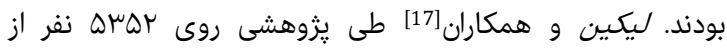

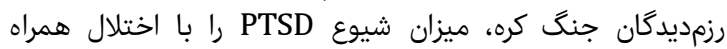

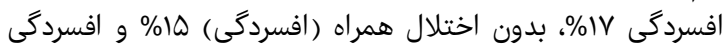

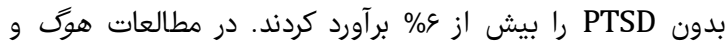

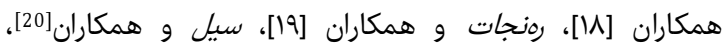

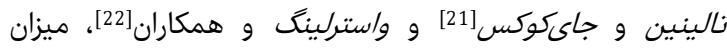

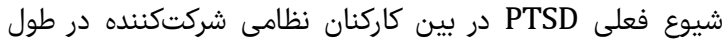

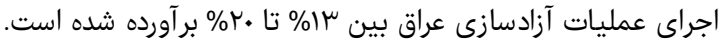

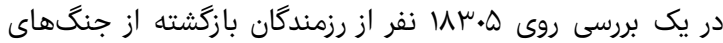

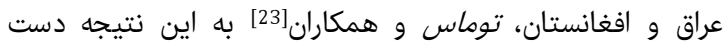

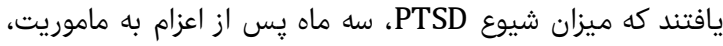

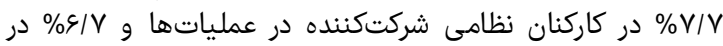

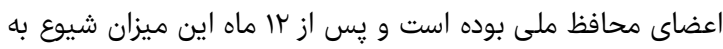

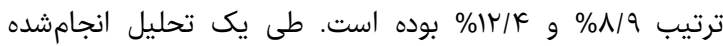

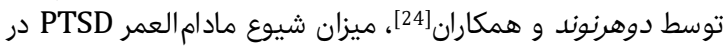

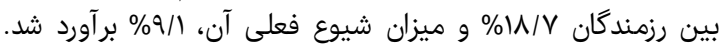

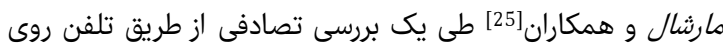

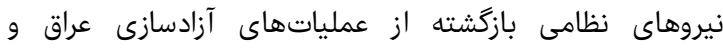

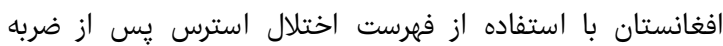

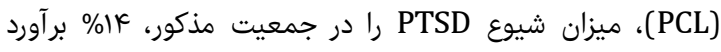

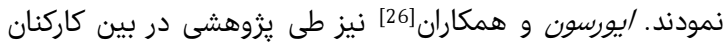

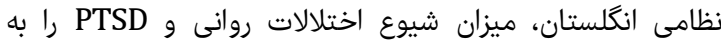

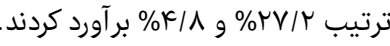

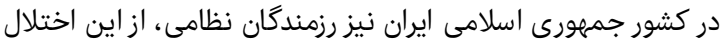

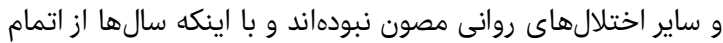

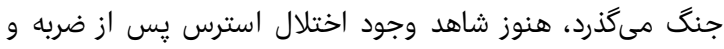

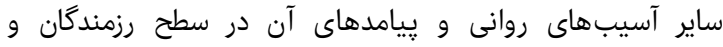

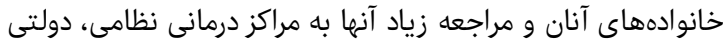

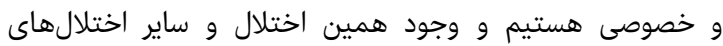

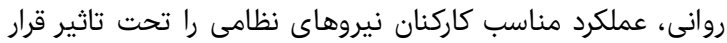

Volume 9, Issue 1, Winter 2017 
استرس يس از ضربه (PCL-M-5) با استفاده از ضريب آلفاى

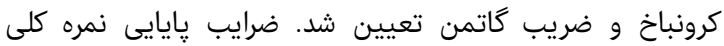

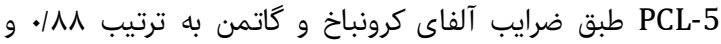

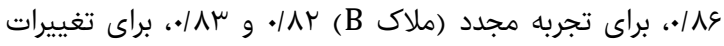

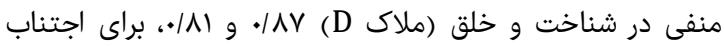

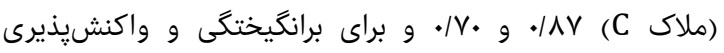

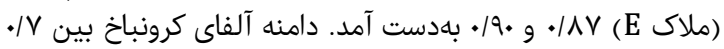

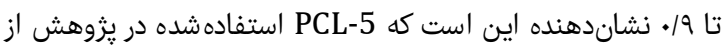

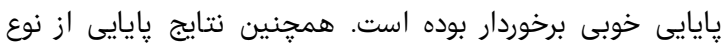

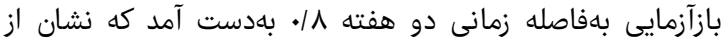

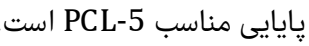

r- مقياس متخصص اجراى ساختاريافته اختلال استرس يس إن ازي

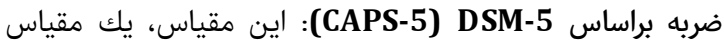

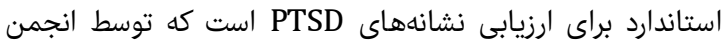

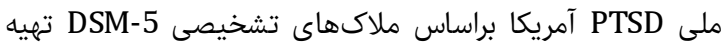

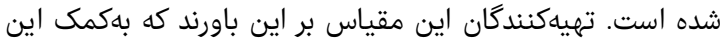

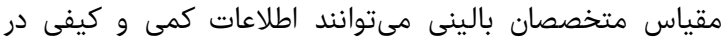

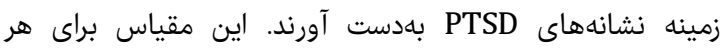

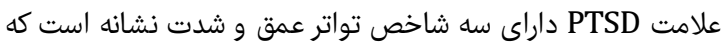

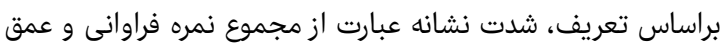

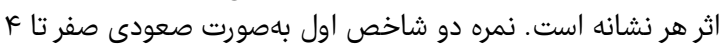

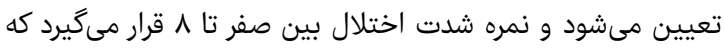

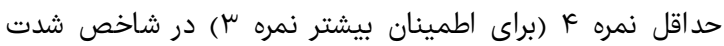

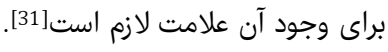

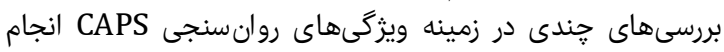

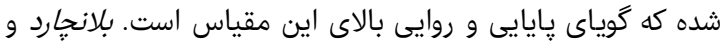

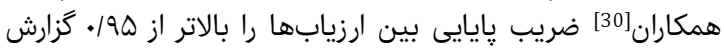

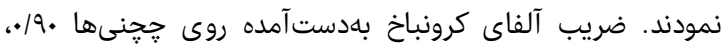

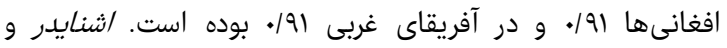

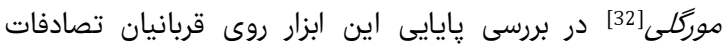

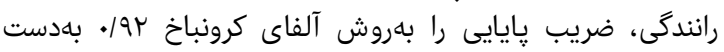

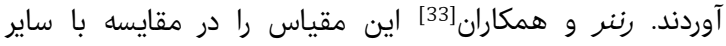
ابزارهاى سنجش علايم PTSD داراى بيشترين ضريب تشخيص

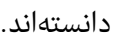

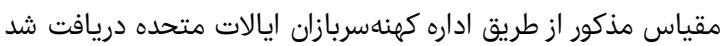

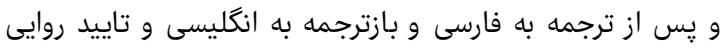

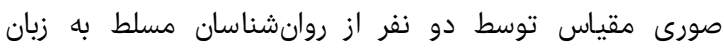

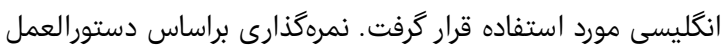

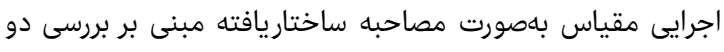

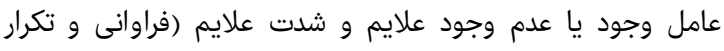

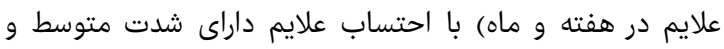

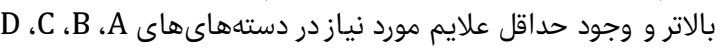

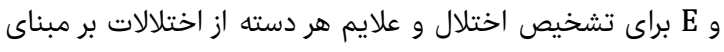

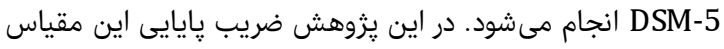

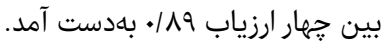

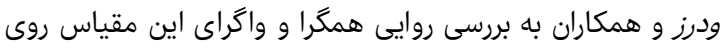

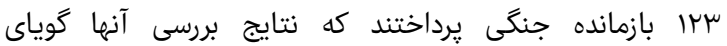

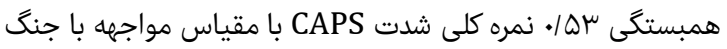

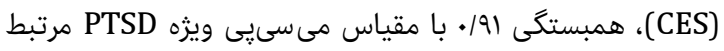

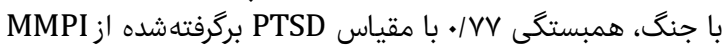

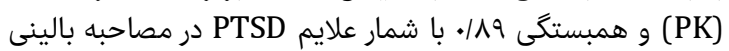

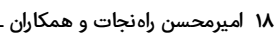

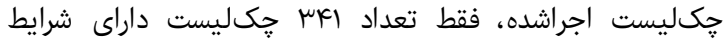

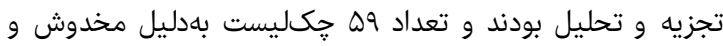

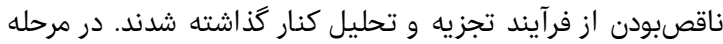
بعدى شركتكنندكانى كه نمره آنها در

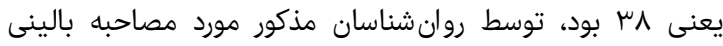
براساس CAPS-5 (مقياس متخصص اجراى ساختاريافته اختلال

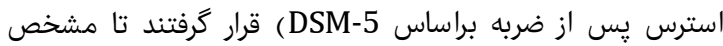

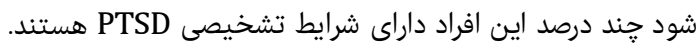

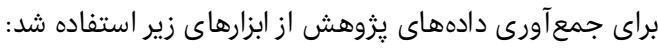

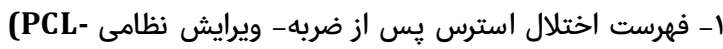

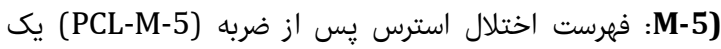

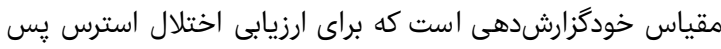

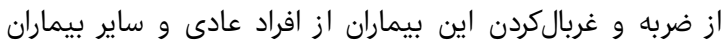

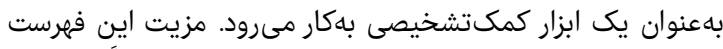

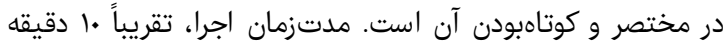

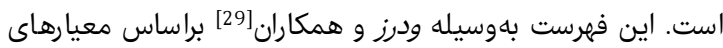

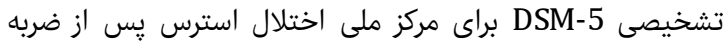

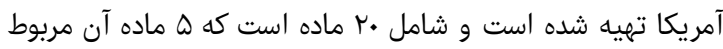

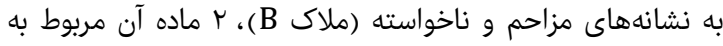

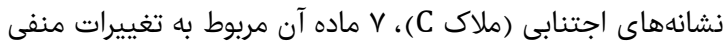

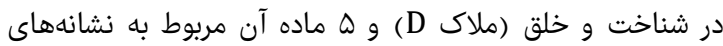

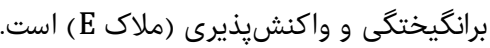

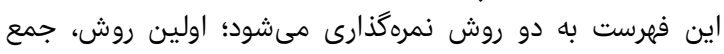

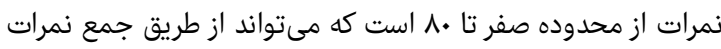

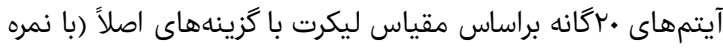

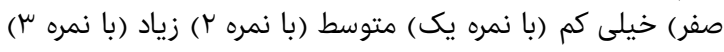

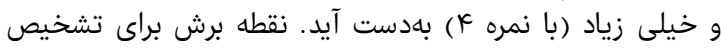
PTSD

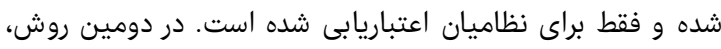

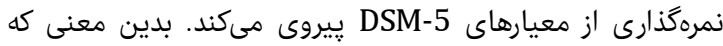

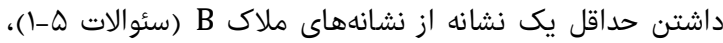

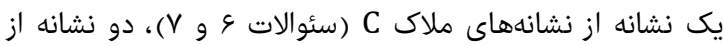

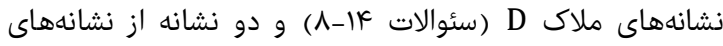

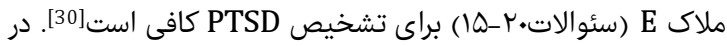

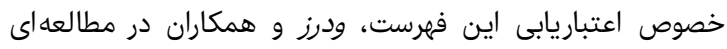

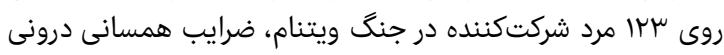

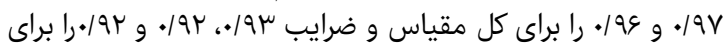

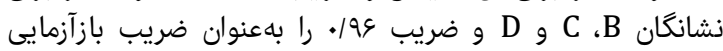

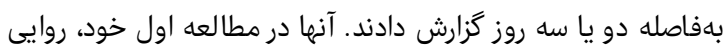

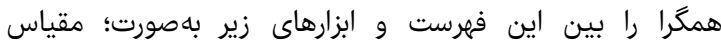

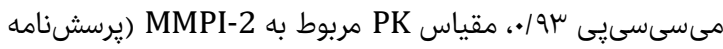

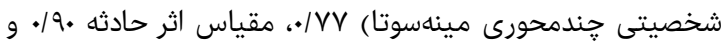

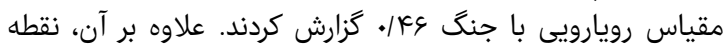

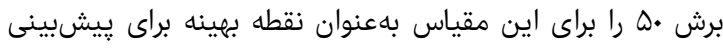

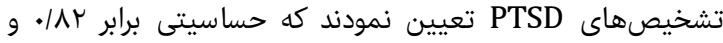

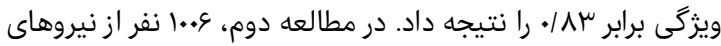

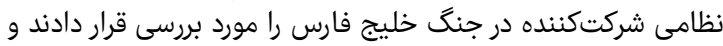

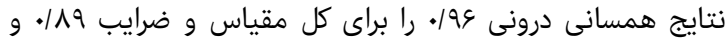

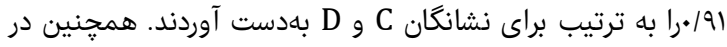

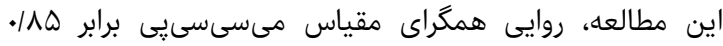

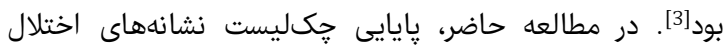




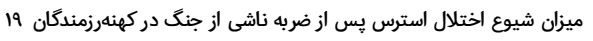

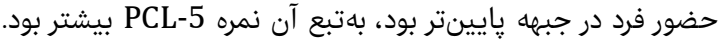

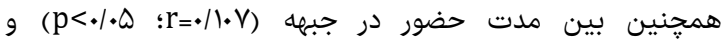

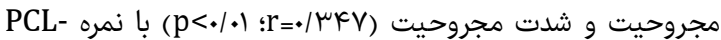

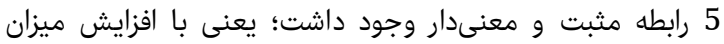

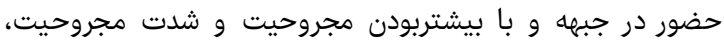

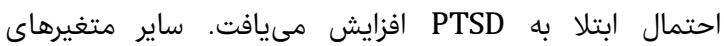

جمعيتشناختى ارتباط معنىدارى با ابتلا به PTSD نداشئ Pائند.

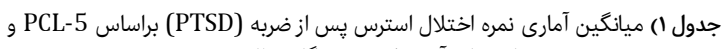

\begin{tabular}{|c|c|c|c|}
\hline حداكثر & حداقل & ميانگين & شاخص \\
\hline Vr & - & $\mu \Lambda / .9 \pm Y \mid / \Lambda$. & 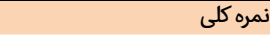 \\
\hline r. & - & 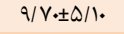 & خردهمقياس تجربه مجدد \\
\hline$\wedge$ & - & $\mu / V \cdot \pm r / r \cdot$ & خردممقياس اجتناب \\
\hline ru & - & $\mid \pi / \kappa_{ \pm} \pm V / \mu$. & تغييرات منفى در شناخت وخُلق \\
\hline r. & - & $|r / r g \pm I| / r V$ & برانكيختكى و واكنشيذيرى \\
\hline
\end{tabular}

همجنين سا\% واريانس مربوط به PTSD، توسط عوامل

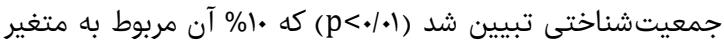

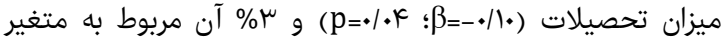

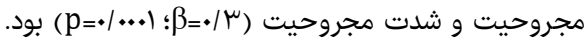

بحث

يزوهش حاضر با هدف شناسايى ميزان و علايم PTSD و آثار

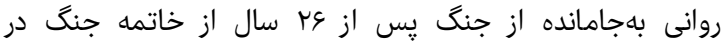

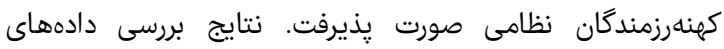

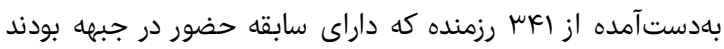

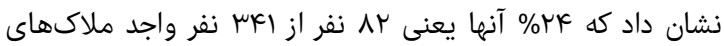

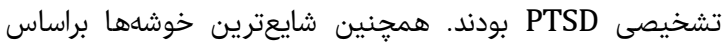

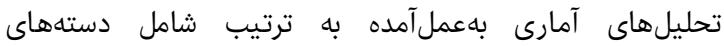

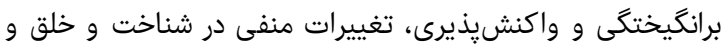

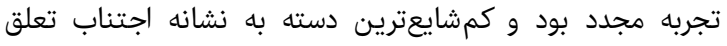
داشت.

در مورد دلايل و تبيينهاى احتمالى اين ميزان از شيوع و تفاوت

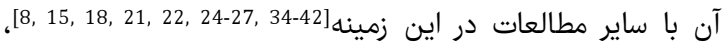

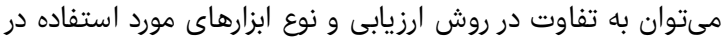

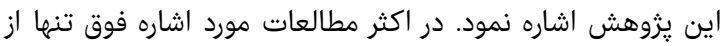

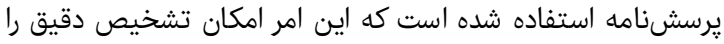

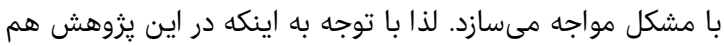

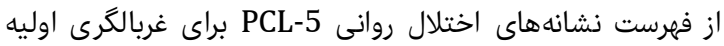

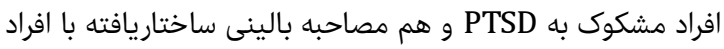

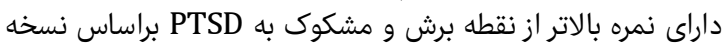

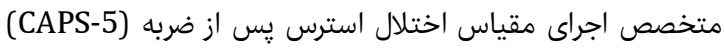

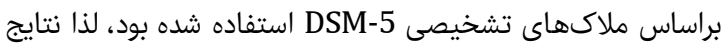

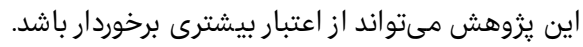

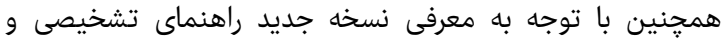

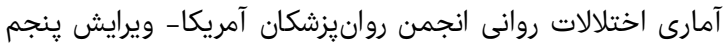

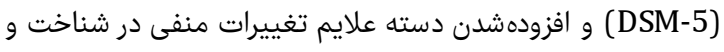

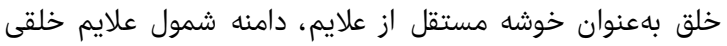

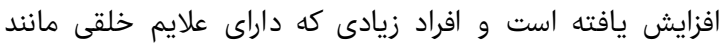

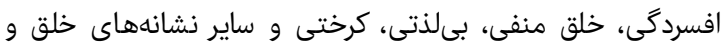

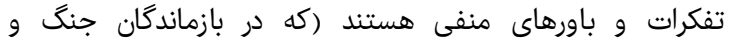

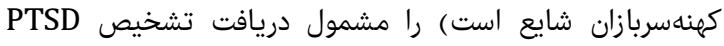

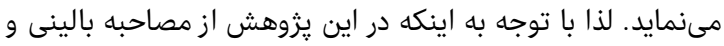

ساختاريافته براى اختلالهاى محور يك (SICD) بود. اين مقياس

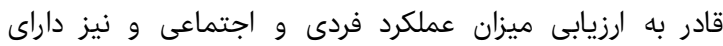

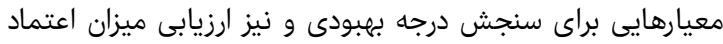

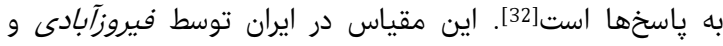

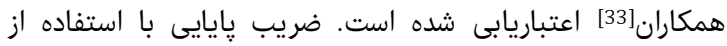

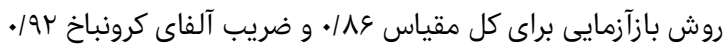

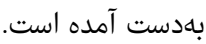

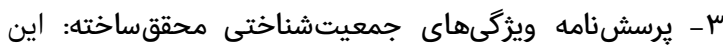

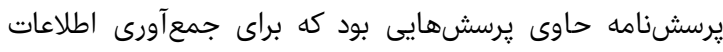

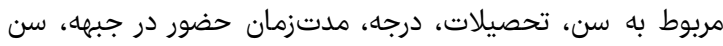

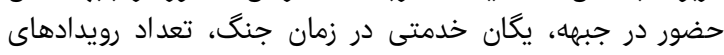

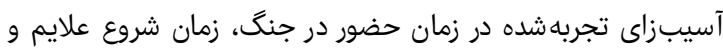

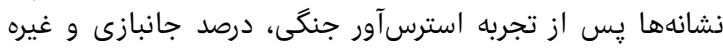

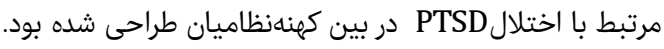

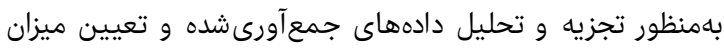

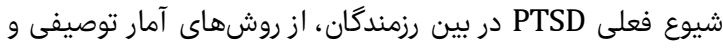

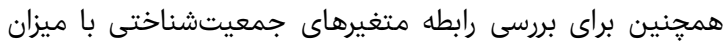

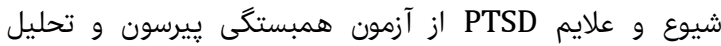
ركرسيون همزمان استفاده شد.

يافتهها

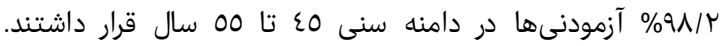

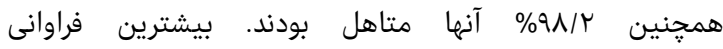

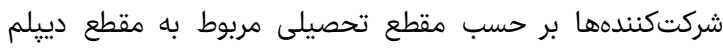

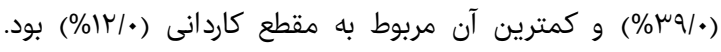

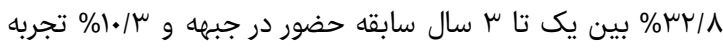

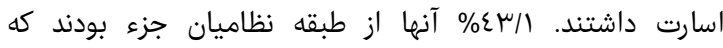

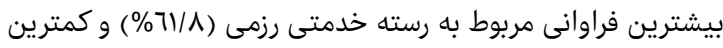

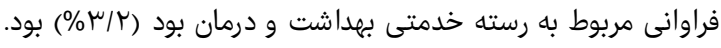

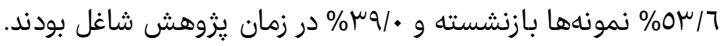

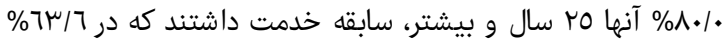

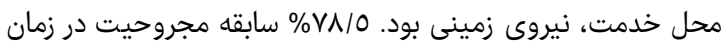

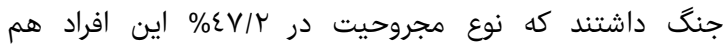

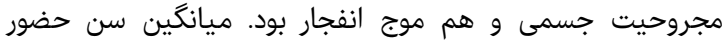

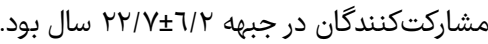

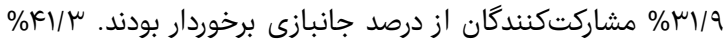

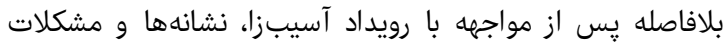

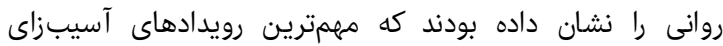

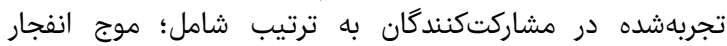

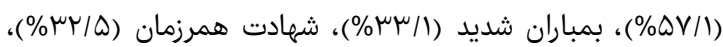

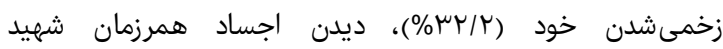

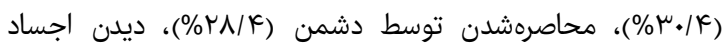

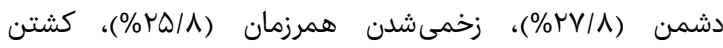

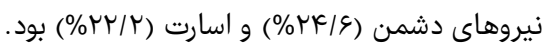

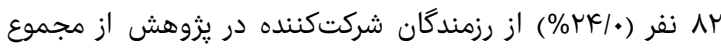

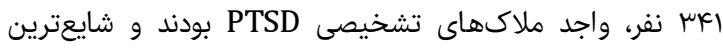

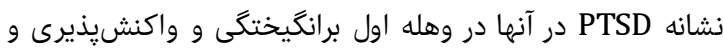

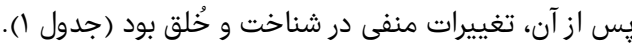

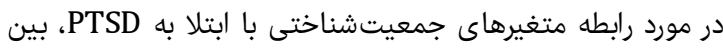

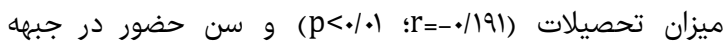

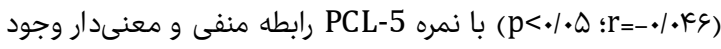

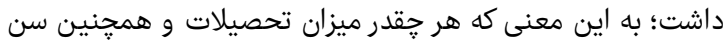


افراد مبتلا به PTSD دنيا را بهصورت كاملاً خطرناك درك نمايند.

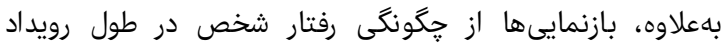

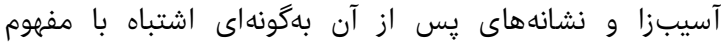

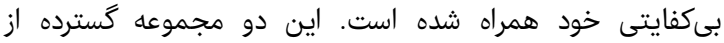

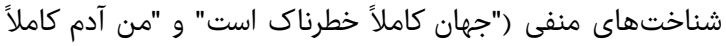

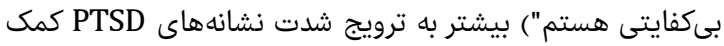

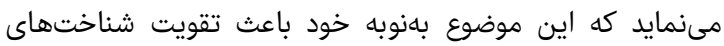
نادرست و منفى مىشود.

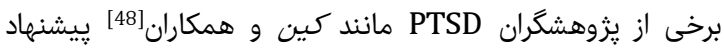

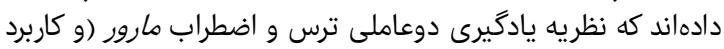

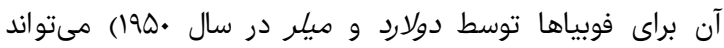

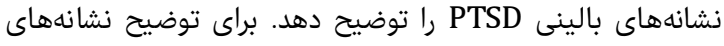

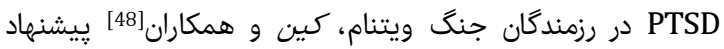

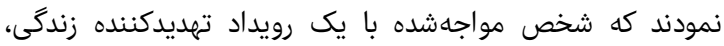

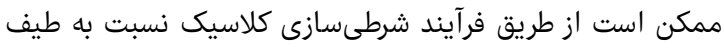

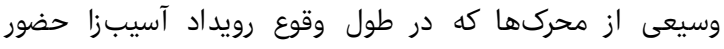

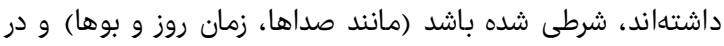

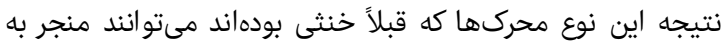

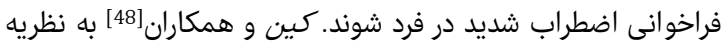

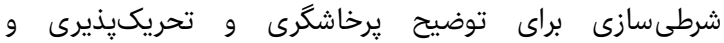

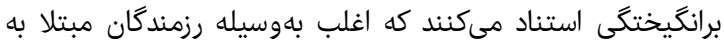

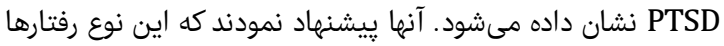

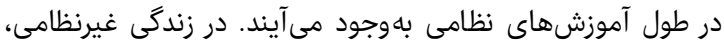

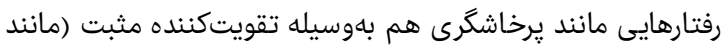

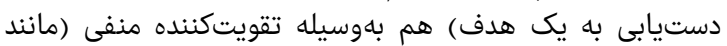

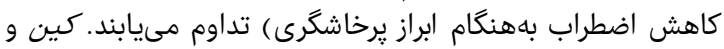

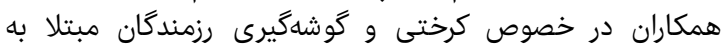

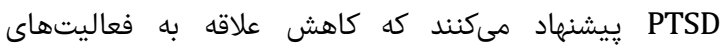

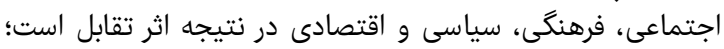

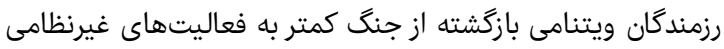

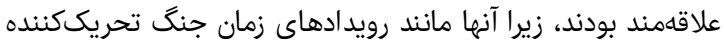

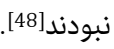
از ديكر نتايج اين يزوهش ارتباط معنىدار متغيرهاى

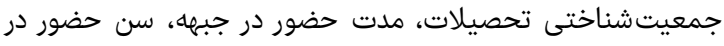

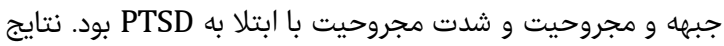

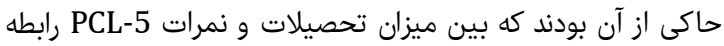

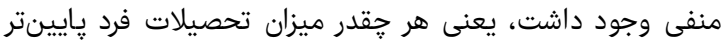

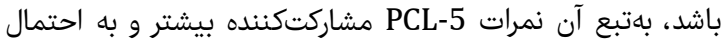

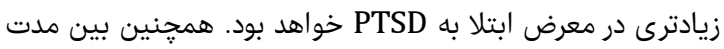

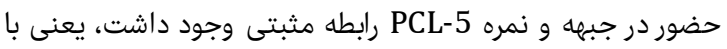

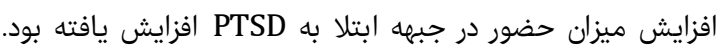

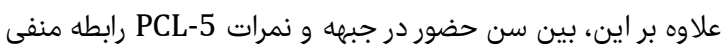

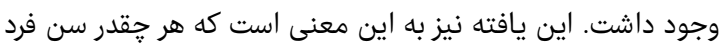

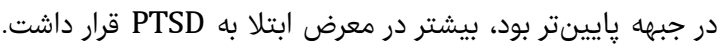

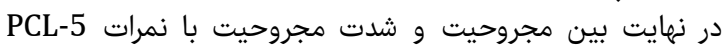

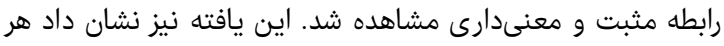

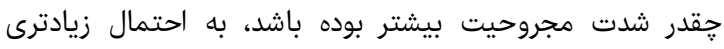
رزمنده ارتشى در معرض ابتلا به PTSD بوديث بوده است.

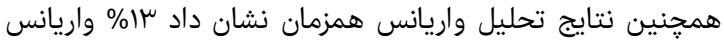

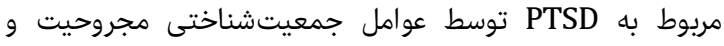

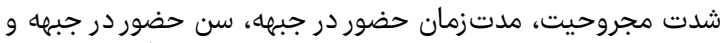
ميزان تحصيلات تبيين مىشود كه اين يافته تقريباً با مطالعاتى دردي
يرسشنامه براساس DSM-5 استفاده شد، افزايش ميزان شيوع در

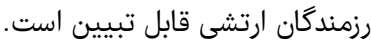

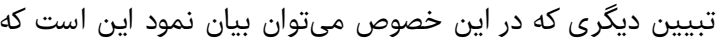

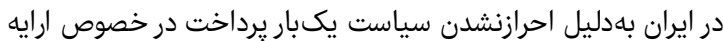

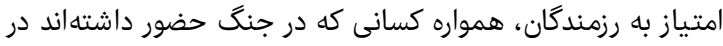

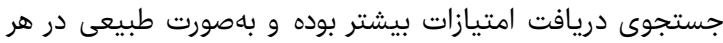

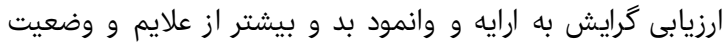

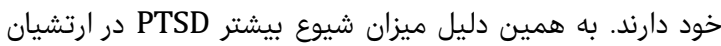

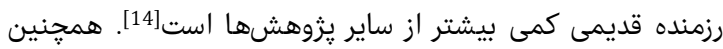

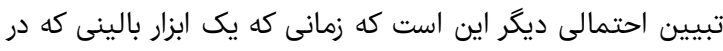

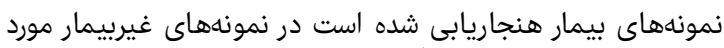

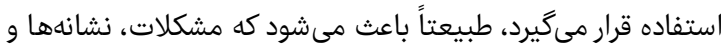

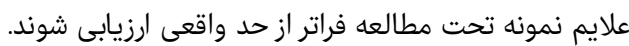

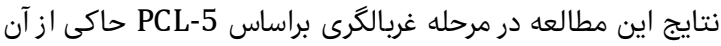

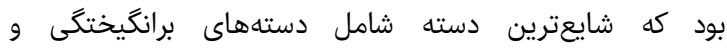

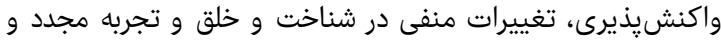

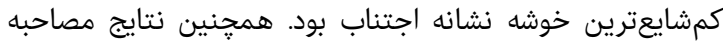

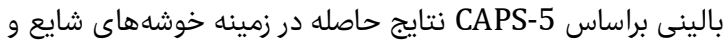

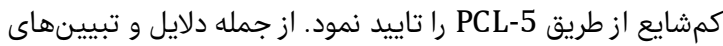

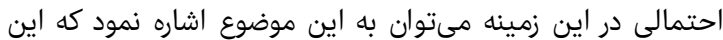

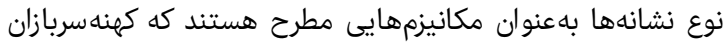

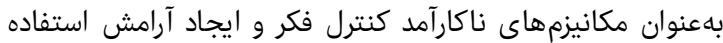

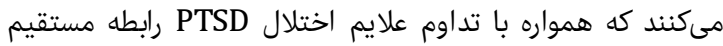

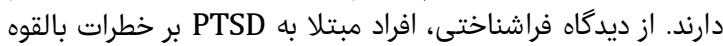

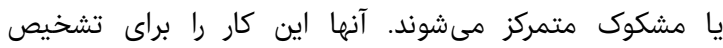

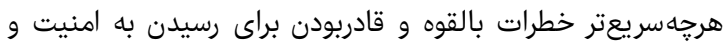

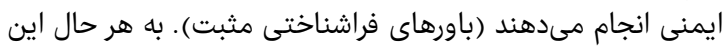

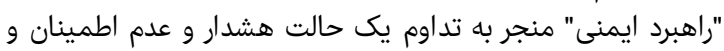

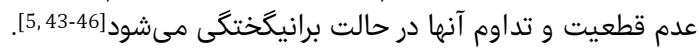

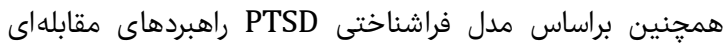

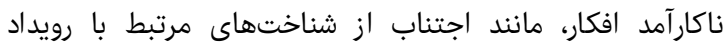

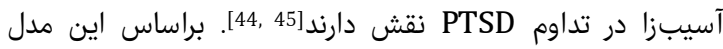

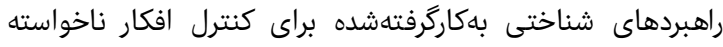

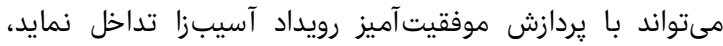

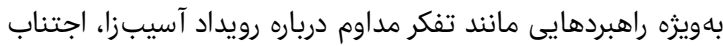

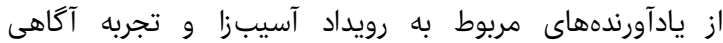

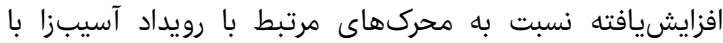

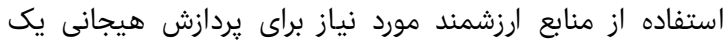

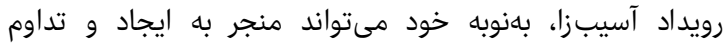

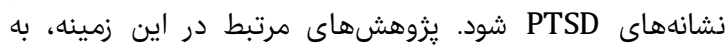

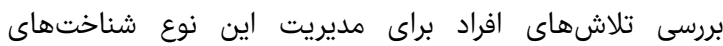

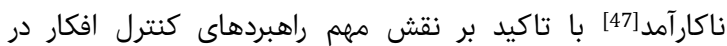

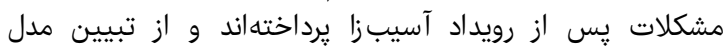

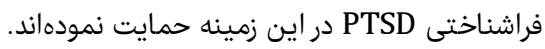

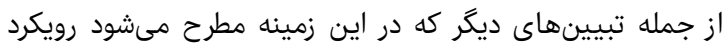

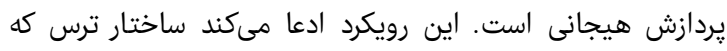

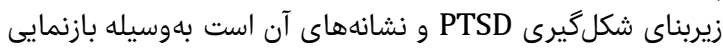

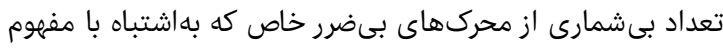

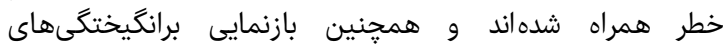

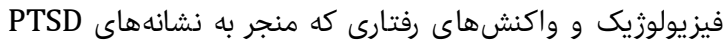

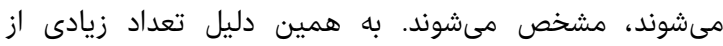

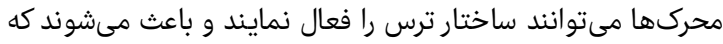

فصل نامه علمى - يخروهشى طب جانباز 


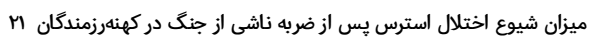

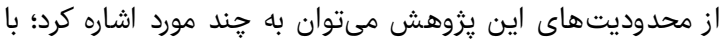

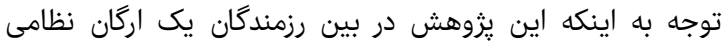

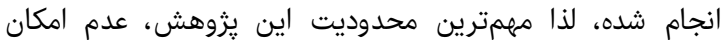

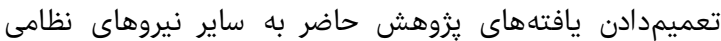

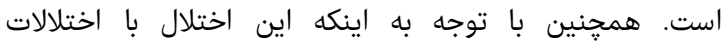

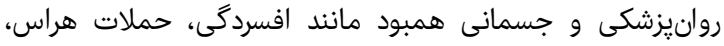

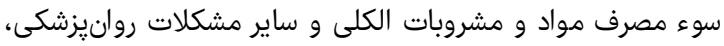

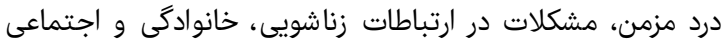

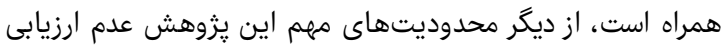

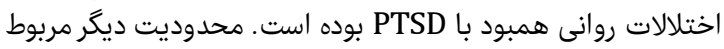

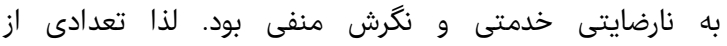

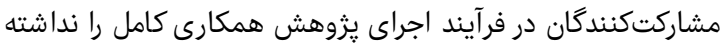

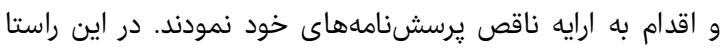

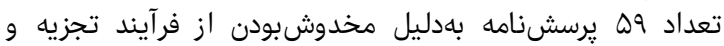

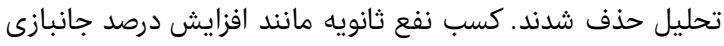

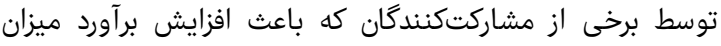

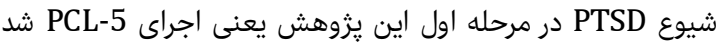

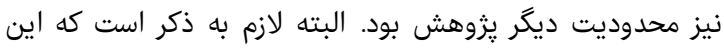

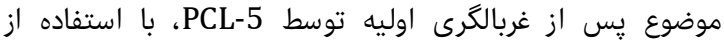

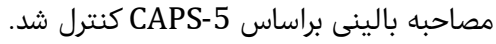

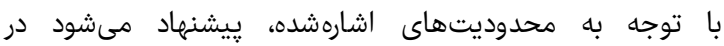

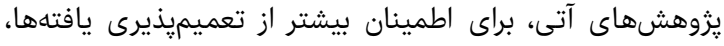

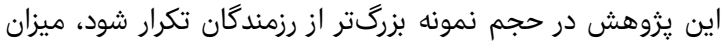

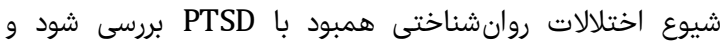

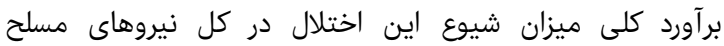

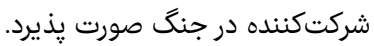

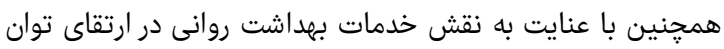

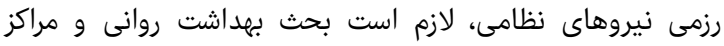

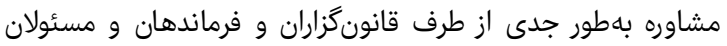

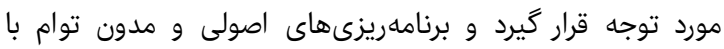

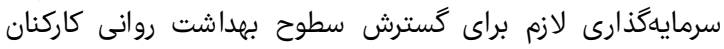

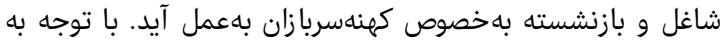

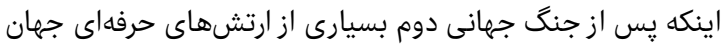

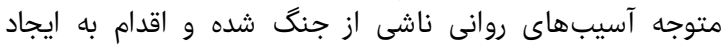

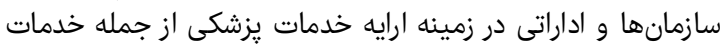

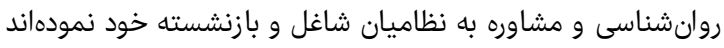

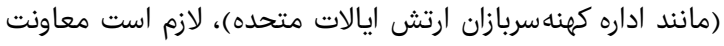

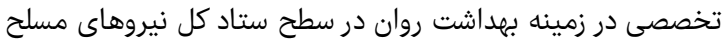

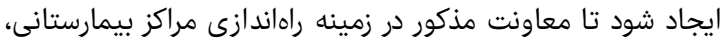

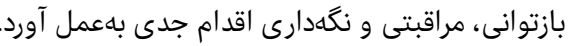

\section{نتيجه}

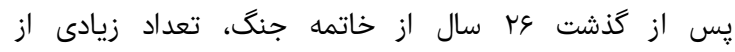

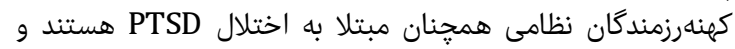

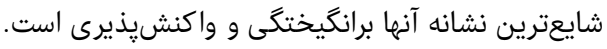

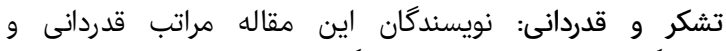

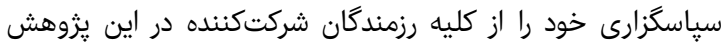

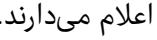
تاييديه اخلاقى: اين مطالعه در كميته اردان اخلاق دانشگاه وابسته به

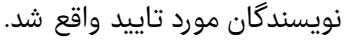

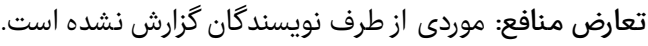

كه در اين زمينه در بين كهنهسربازان انجام شده ,24, 18, 20-22, 24- كهوان

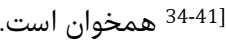

از جمله تبيينهاى قابل طرح در اين زمينه، مىتوان به موارد ذيل

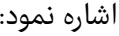

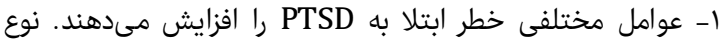

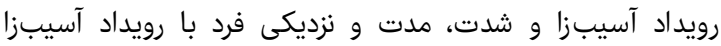

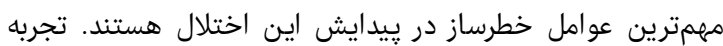

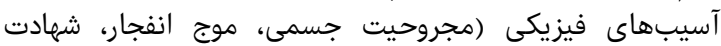

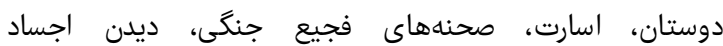

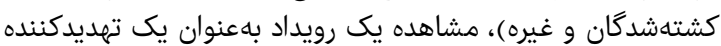

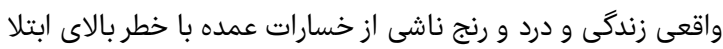

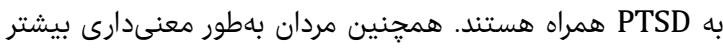

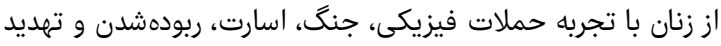

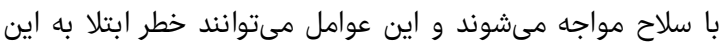

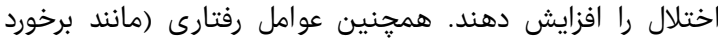

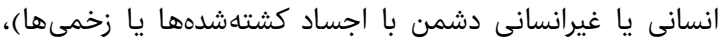

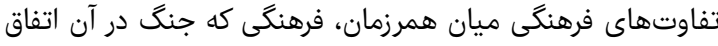

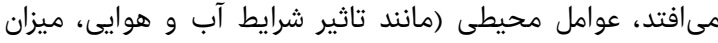

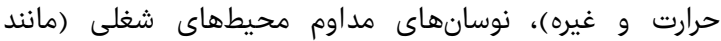

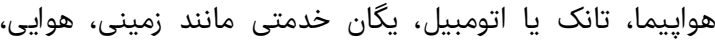

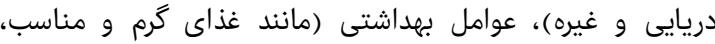

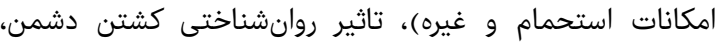

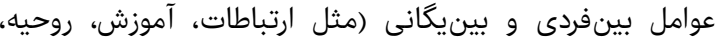

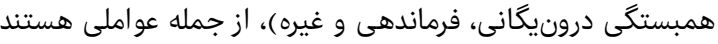

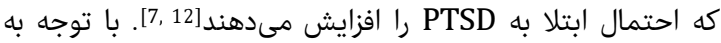

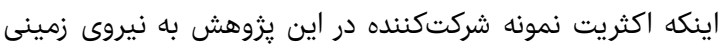

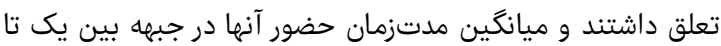

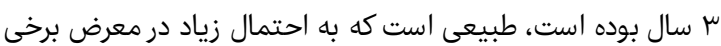

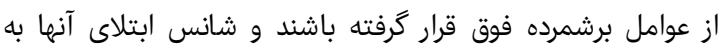
PTSD

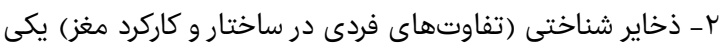

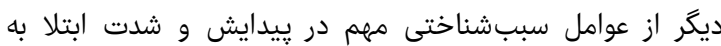

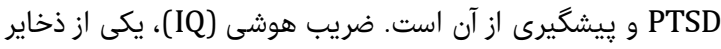

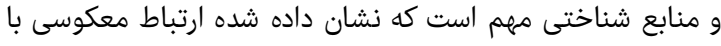

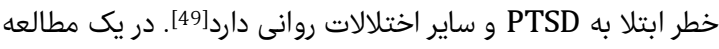

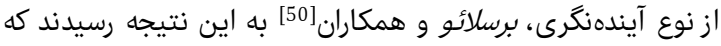

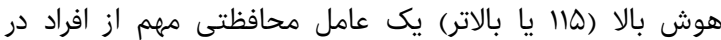

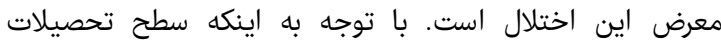

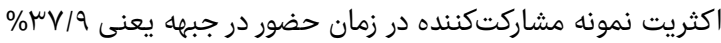

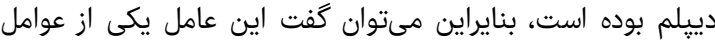

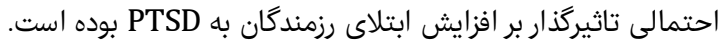

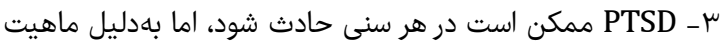

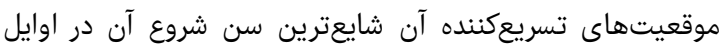

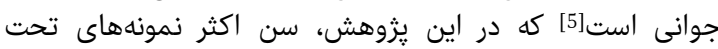

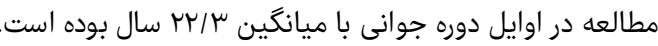

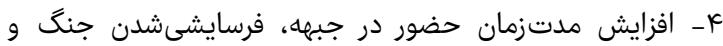

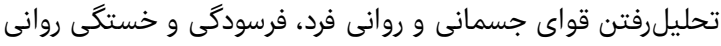

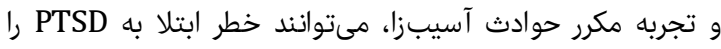

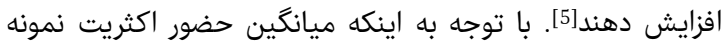

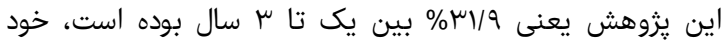

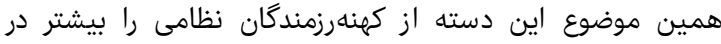
معرض تشخيص PTSD قرار داده است. 
13- Coleman P. Flashback: Post traumatic stress disorder, suicide, and the lessons of war. $1^{\text {st }}$ edition. Boston: Beacon Press; 2007.

14- Dabaghi P. Cognitive behavior therapy of post traumatic stress disorder - treatment guide for clinicians. Tehran: Jahad Daneshgahi Publication; 2008. [Persian] 15- Seal KH, Bertenthal D, Miner CR, Sen S, Marmar C. Bringing the war back home: mental health disorders among 103,788 US veterans returning from Iraq and Afghanistan seen at Department of Veterans Affairs facilities. Arch Intern Med. 2007;167(5):476-82.

16- Smith TC, Wingard DL, Ryan MA, Kritz-Silverstein D, Slymen DJ, Sallis JF. PTSD prevalence, associated exposures, and functional health outcomes in a large, population-based military cohort. Public Health Rep. 2009;124(1):90-102.

17- Likin JF, Creamer MC, Sim MR, McKenzie DP. Comorbidity of PTSD and depression in Korean War veterans: prevalence, predictors, and impairment. J Affect Disord. 2010;125(1-3):279-86.

18- Hoge CW, Castro CA, Messer SC, McGurk D, Cotting DI, Koffman RL. Combat duty in Iraq and Afghanistan, mental health problems, and barriers to care.N Engl J Med. 2004;351:13-22.

19- Rah Nejat AM, Bahmin Gh, Sajadian SR, Donyavi V. epidemiological study of psychological disorders in one of the ground units military forces of Islamic Republic of Iran. J Mil Psychol. 2011;2(6):27-36. [Persian]

20- Seal KH, Metzler TJ, Gima KS, Bertenthal D, Maguen S, Marmar CR. Trends and risk factors for mental health diagnoses among Iraq and Afghanistan veteransusing Department of Veterans Affairs health care, 2002-2008. Am J Public Health. 2009;99(9):1651-8.

21- Tanielian TL, Jaycox L. Invisible wounds of war: Psychological and cognitive injuries, their consequences, and services to assist recovery. Arlington, VA: RAND Corporation; 2008.

22- Vasterling JJ, Proctor SP, Friedman MJ, Hoge CW, Heeren T, King LA, et al. PTSD symptom increases in Iraq-deployed soldiers: Comparison with no deployed soldiers and associations with baseline symptoms, deployment experiences, and post deployment stress. J Trauma Stress. 2010;23(1):41-51.

23- Thomas JL, Wilk, JE, Riviere LA, McGurk D, Castro CA, Hoge CW. Prevalence of mental health problems and functional impairment among active component and National Guard soldiers 3 and 12 months following combat in Iraq. Arch Gen Psychiatry. 2010;67(6):614-23. 24- Dohrenwend BP, Turner JB, Turse NA, Adams BG, Koenen KC, Marshall R. The psychological risks of Vietnam for U.S. veterans: A revisit with new data and methods. Science. 2006;313(5789):979-82.

25- Marshall GN, Schell TL, Miles JN. Ethnic differences in posttraumatic distress: Hispanics' symptoms differ in kind and degree. J Consult Clin Psychol. 2009;77(6):1169-78.

26- Iversen AC1, van Staden L, Hughes JH, Browne T, Hull $\mathrm{L}$, Hall J, et al. The prevalence of common mental disorders and PTSD in the UK military: Using data from a clinical interview-based study. BMC Psychiatry. 2009;9:68.

27- Donyavi V, Shafighi F, ROUHANI SM, Hosseini S, Kazemi J, Arghanoun S, et al. The prevalence of ptsd in conscript and official staff of earth force in tehran during 2005-6. Ann Mil Health Sci Res. 2007;5(1):1121-5. [Persian]

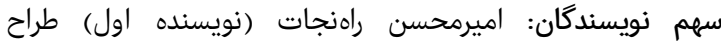

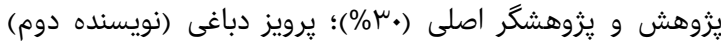

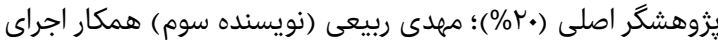

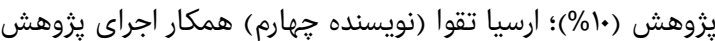

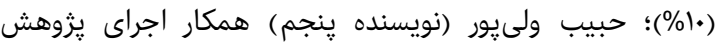

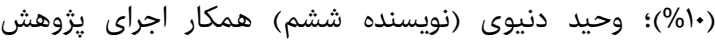

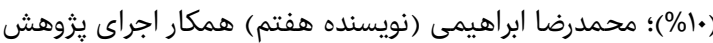

منابع مالى: اين مطالعه بخشى از يك كار يزوهشى است كه با

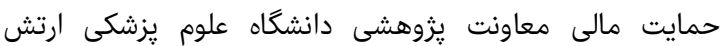

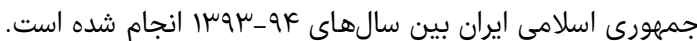

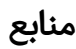

1- Breslau N1, Davis GC, Andreski P, Peterson E. Traumatic events and posttraumatic stress disorder in an urban population of young adults. Arch Gen Psychiatry. 1991;48(3):216-22.

2- Yehuda Rachel. Treating trauma survivors with PTSD. Washington, DC: American Psychiatric Publishing; 2002.

3- Rahnejat AM, Rabiei M, Salimi SH, Fathi Ashtiani A, Donyavi $\mathrm{V}$, et al. Causal metacognitive model war-related chronic posttraumatic stress disorder. Iran J Psychiatry Clin Psycol. 2015;20(4):317-25. [Persian]

4- Salimi SH, Rahnejat AM, Rabiei M, Fathi Ashtiani A, Dabaghi $\mathrm{P}$, Donyavi. Review and analysis of the Meta cognitive therapy of post traumatic stress disorder: A systematic review of studies. J Nurs Physician War. 2014;2(2):38-45. [Persian]

5- Rahnejat AM. Comparative effectiveness of metacognitive therapy and prolonged exposure therapy on the reduction of chronic post traumatic stress disorder caused by the war [Dissertation]. Tehran: Baqiyatallah University of Medical Science; 2014. [Persian]

6- Zungu LI. Prevalence of post-traumatic stress disorder in the South African mining industry and outcomes of liability claims submitted to Rand Mutual Assurance Company. Occup Health South Afr. 2013;19(2):22-6.

7- Kessler RC, Sonnega A, Bromet E, Hughes M, Nelson CB. Posttraumatic stress disorder in the National Comorbidity Survey. Arch Gen Psychiatry. 1995;52(12):1048-60.

8- Kulka, RA, Schlenger WE, Fairbank JA, Hough RL, Jordan BK, Marmar CR, et al. Trauma and the vietnam war generation: Report of findings from the national vietnam veterans readjustment study. $1^{\text {st }}$ edition. New York: Brunner Mazel Publishers; 1990

9- Prigerson HG, Maciejewski PK, Rosenheck RA. Population attributable fractions of psychiatric disorders and behavioral outcomes associated with combat exposure among US men. Am J Public Health. 2002;92(1):59-63.

10- Sundin J, Fear NT, Iversen ARJ, Rona RJ, Wessely S. PTSD after deployment to Iraq: Conflicting rates, conflicting claims. Psychol Med. 2010;40(3):367-82.

11- Paulson DS, Krippner S. Haunted by combat: Understanding PTSD in war veterans including women, reservists, and those coming back from Iraq. London: Praeger Security International; 2007.

12- Kennedy $\mathrm{CH}$, Zillmer EA. Military psychol: Clinical and operational applications. New York: Guilford Press; 2006. 
ميزان شيوع اختلال استرس يّ ازضربه ناشى از جنگ در كهنهرزمندكان سب ,

39- Kang HK, Natelson BH, Mahan CM, Lee KY, Murphy

FM. Post-traumatic stress disorder and chronic fatigue syndrome-like illness among Gulf War veterans: A population-based survey of 30,000 veterans. Am J Epidemiol. 2003;157(2):141-8.

40- Hotopf M1, Hull L, Fear NT, Browne T, Horn O, Iversen $\mathrm{A}$, et al. The health of UK military personnel who deployed to the 2003 Iraq war: A cohort study. Lancet. 2006;367(9524):1731-41.

41- Kozarić-Kovačić, D, Borovečki A. Prevalence of psychotic co morbidity in combat-related post-traumatic stress disorder. Mil Med. 2005;170(3):223-6.

42- Taheri S, Shabani A. Conceptual and practical principles in designing healing gardens for veterans with PTSD with a focus on reducing stress- a narrative review. J Mil Med. 2016;18(3):230-41. [Persian]

43- Simon M. Meta cognitive therapy and other cognitive - behavioral treatments for posttraumatic stress disorder. Verhaltenstherapie. 2010;20:86-92.

44- Wells A, Sembi S. Met cognitive therapy for PTSD: A preliminary investigation of a new brief treatment. J Behav Ther Exp Psychiatry. 2004;35(4):307-18.

45- Wells A, Welford M, Fraser J, King P, Mendel E, Wisely J, et al. Chronic PTSD Treated with Meta cognitive Therapy: An Open Trial. Cogn Behav Pract. 2008;15(1):85-92.

46- Bennett SA, Beck JG, Clapp JD. Understanding the relationship between posttraumatic stress disorder and trauma cognitions: The impact of thought control strategies. Behav Res Ther. 2009;47(12):1018-23.

47- Reynolds M, Well A. The Thought Control Questionnaire--psychometric properties in a clinical sample, and relationships with PTSD and depression. Psychol Med. 1999;29(5):1089-99.

48- Keane TM, Zimering RT, Caddell JM. A behavioral formulation of posttraumatic stress disorder. Behav Ther. 1985;8(1):9-12.

49- Barnett JH, Salmond CH, Jones PB, Sahakian BJ. Cognitive reserve in neuropsychiatry. Psychol Med. 2006;36(8):1053-64.

50- Breslau N1, Lucia VC, Alvarado GF. Intelligence and other predisposing factors in exposure to trauma and posttraumatic stress disorder: A follow-up study at age 17 years. Arch Gen Psychiatry. 2006;63(11):1238-45.
28- Tavallaie SA, Assari SH, Najafi M, Habibi M, Ghanei M. Study of sleep quality in chemical-warfare-agents exposed veterans. J Mil Med. 2005;6(4) :241-48. [Persian]

29- Rahnejat AM, Rabiei M, Salimi SH, Fathi-Ashtiani A, Donyavi V, Mirzai J. The role of thought control strategies on the symptoms of chronic Post-Traumatic Stress Disorders caused by war. J Behav Sci. 2015;8(4):347-54. [Persian]

30- Blanchard EB, Jones-Alexander J, Buckley TC, Forneris CA. Psychometric properties of the PTSD Checklist (PCL). Behav Res Ther. 1996;34(8):669-73.

31- Hoge CW, Riviere LA, Wilk JE, Herrell RK, Weathers FW. The prevalence of post-traumatic stress disorder (PTSD) in US combats soldiers: A head-to-head comparison of DSM-5 versus DSM-IV-TR symptom criteria with the PTSD checklist. Lancet Psychiatry. 2014;1(4):269-77.

32- Weathers FW, Marx BP, Friedman MJ, Schnurr PP. Posttraumatic stress disorder in DSM-5: New criteria, new measures, and implications for assessment. Psychol Inj Law. 2014;7(2):93-107.

33- Firoozabadi A, Asgharnejad Farid AA, Mirzaei J, Shareh H. Normalization of Clinician Administered PTSD Scale-version 1 (CAPS-1) for Psychological Effects due to War. Iran J Psychiatry Clin Psycol. 2010;15(4):334-42. [Persian]

34- Institute of Medicine (IOM). Treatment for posttraumatic stress disorder in military and veteran populations: Initial assessment. Washington, DC: The National Academies Press; 2012.

35- US Centers for Disease Control. Health status of Vietnam veterans. I. Psychosocial characteristics. The Centers for Disease Control Vietnam Experience Study. JAMA. 1988;259(18):2701-7.

36- Port CL, Engdahl B, Frazier P. A longitudinal and retrospective study of PTSD among older prisoners of war. Am J Psychiatry. 2001;158(9):1474-9.

37- Richardson LK, Frueh BC, Acierno R. Prevalence estimates of combat-related PTSD: A critical review. Aust N Z J Psychiatry. 2010;44(1):4-19.

38- Solomon Z, Dekel R. Posttraumatic stress disorder and posttraumatic growth among Israeli ex-pows. J Trauma Stress. 2007;20(3):303-12. 